\title{
LA GEOLOGÍA, VISTA DESDE LA FILOSOFÍA
}

\author{
Luis Camacho
}

\author{
Escuela de Filosofía, Universidad de Costa Rica \\ Presidente, Asociación Costarricense de Filosofía \\ Apartado 388-2050, Costa Rica \\ lcamacho@cariari.ucr.ac.cr
}

(Recibido: 12/07/06; aceptado: 20/11/2008)

\begin{abstract}
Philosophy of science took physics as the model for all sciences, at the expense of other sciences like geology. Immune from criticism and forgotten by both critics and defenders of scientific theories and institutions, geology enjoys a peculiar position which in fact has been an obstacle for a philosophical discussion on object, methods and types of reasoning. A failed prediction of an earthquake in Costa Rica is taken as an example of an issue worth analyzing.

Key words: geology, physics, philosophy of science, theory, method.

RESUMEN: La filosofía de la ciencia tomó la física como modelo de todas las ciencias, en perjuicio de otras ciencias como la geología. Inmune a las críticas y olvidada por los defensores de teorías e instituciones científicas, la geología disfruta de una posición que de hecho ha sido un obstáculo para la discusión filosófica sobre el objeto, métodos y tipos de razonamiento. La predicción fallida de un terremoto en Costa Rica se toma como ejemplo de un asunto digno de análisis.

Palabras clave: geología, física, filosofía de la ciencia, teoría, método.
\end{abstract}

\section{INTRODUCCIÓN}

La filosofía de la ciencia tal como la conocemos en nuestros días, es decir como una disciplina universitaria con respaldo institucional, nació en 1895 en la Universidad de Viena con la creación de una cátedra de filosofía de las ciencias inductivas ocupada primero y hasta 1901 por el físico Ernst Mach (1838-1916), quien también es considerado por muchos el primer filósofo de la ciencia en el sentido contemporáneo del término. Destaca este autor entre otras cosas por su obra Análisis de la sensación y su reconstrucción de la física según su propia visión de la ciencia en la obra de 1883 conocida en inglés como The Science of Mechanics, que tuvo una enorme difusión. Mach estaba preocupado por el uso impreciso y hasta contradictorio de términos en la ciencia. Intentó, además, unificar 
todas las ciencias mediante su teoría de la sensación: nos hacemos representaciones mentales del mundo, que nos permiten operar con las ideas sin necesidad de acudir repetidamente a los hechos. $\mathrm{Su}$ deseo de encontrar solución a problemas internos de la ciencia tal como ésta se da en la realidad es típico de la filosofía de la ciencia actual, a diferencia de las reflexiones más generales de épocas anteriores.

Fue también en Viena donde se fundó la Ernst Mach Verein (Fundación Ernst Mach) en 1928, sustento legal del Círculo de Viena, grupo de científicos y filósofos reunidos en torno a Moritz Schlick a partir de 1922 que pasaron a la historia con el nombre colectivo de positivismo lógico. El Círculo de Viena tuvo filiales en Berlín, Praga y varias ciudades polacas. Después del asesinato de Moritz Schlick en 1936 por un estudiante que fue aclamado como héroe por los nazis, los miembros del grupo tuvieron que dispersarse en el extranjero.

Mucho después del inicio de la diáspora, Ernest Nagel (1901-1985) expuso en The Structure of Science (1961) la versión más acabada de la filosofía de la ciencia tal como fue construida por los positivistas lógicos y sus sucesores, los filósofos analíticos. Pero desde años antes había habido discrepancias en conexión con las tesis principales del Círculo (sobre todo en lo relacionado con la verificación), y por eso muchos de los filósofos de la ciencia posteriores a la muerte de Schlick se definen en gran medida por su grado de alejamiento del positivismo lógico, tomado como punto de referencia. Karl R. Popper (1902-1994), Thomas S. Kuhn (1922-1996), Imre Lakatos (1922-1974) , Paul Feyerabend (19241994) y otras luminarias menores escribieron largo y tendido sobre la naturaleza de la ciencia, la relación entre ciencia y sociedad y el papel de teorías e hipótesis, en discusión con el Círculo de Viena y entre sí. Popper defendió el método hipotético-deductivo, Kuhn analizó la historia de la ciencia con las nociones de paradigma y revolución, Lakatos introdujo la idea de programas de investigación (cada uno con la metodología que prefiera, pero todos con transparencia hacia los demás), y Feyerabend se volvió famoso con su anarquismo metodológico ("todo vale").
Un breve examen de la producción de estos autores muestra un marcado sesgo hacia la física. Los miembros del Círculo de Viena y de sus sucursales - los más connotados fueron Moritz Schlick (1882-1936), Rudolf Carnap (1891-1970), Otto von Neurath (1882-1945), Carl Hempel (19051997) y Hans Reichenbach (1891-1953) - rara vez hablaron de otras ciencias. Nagel dedica una sección de su libro a las ciencias sociales (en particular a la historia) pero niega que sean ciencias con características profundas que las separen de la física. Popper discutió con Teodoro Adorno sobre la naturaleza de las ciencias sociales, pero sostiene que comparten el mismo método de la física. También desde el inicio hubo algunas referencias a la biología, incluso en escritos tempranos del fundador del Círculo, Moritz Schlick.

\section{Una ciencia olvidada por la filosofía}

¿Y la geología? En un recuento que hemos hecho de 20 libros de filosofía de la ciencia de los autores mencionados, no hemos encontrado ni una sola referencia a la geología ni tampoco a los fundadores de esta ciencia (Abraham Gottlob Werner, James Hutton, Charles Lyell). En un examen de 107 números de la revista Philosophy of Science (órgano oficial de la asociación internacional correspondiente), entre los años 1960 y 1995 , las únicas referencias a la geología que pudimos localizar son las reseñas de dos libros : el de David B. Kitts , The Structure of Geology (Dallas: Southern Methodist Press, 1977) y el de Rachel Laudan titulado From Mineralogy to Geology: The Foundations of a Science, 16501830 (Chicago University Press, 1987). La primera reseña aparece en el número correspondiente a marzo de 1979 y la segunda en el de junio de 1990. La autora de la primera reseña, Rachel Laudan, es también la autora del libro reseñado en la segunda. Justamente en esa primera reseña encontrada la autora confirma la impresión que nos llevó a hacer el recuento, pues empieza señalando el olvido en que ha caído la geología entre los filósofos de la ciencia a pesar de sus éxitos recientes y de que grandes científicos del siglo XIX, sobre todo el físico William Whewell y el 
astrónomo John Herschel, no solo contribuyeron a su desarrollo sino que reflexionaron sobre su naturaleza. De modo que al tratar de confirmar nuestra hipótesis de que la geología ha sido olvidada ¡la primera referencia que encontramos a la geología empieza afirmando que la geología ha sido olvidada!

Esta situación se repite en la historia de la ciencia, otra actividad íntimamente relacionada con la filosofía hasta el punto de que en algunos países son los filósofos y científicos quienes se ocupan de ella, no los historiadores. Un artículo de Mott T.Greene, publicado en Osiris en 1985 resume la situación de la siguiente forma:

La historia de la geología es la más joven de las ramas principales de la historia de la ciencia (...).Esta área empezó a existir en la última década y media y es mucho más joven que la historia de la física, química, biología, astronomía o matemáticas(...) Hay pruebas abundantes de que los historiadores de la ciencia todavía piensan que la historia de la ciencia es ante todo la historia de la física (inorgánica) y/o de la biología (orgánica) (...) Las razones para esta obsesión con la física y la biología no son relevantes aquí, pero sí son sus efectos. [Osiris segunda serie, 1985, 1, pp. 97-116. La traducción es del autor del presente artículo. Osiris es una publicación dedicada a la historia de la ciencia, con ensayos más largos que los aparecidos en Isis, la otra publicación de la misma disciplina].

¿Cuáles son esos efectos? Según Greene, las teorías del cambio científico desarrolladas por filósofos de la ciencia con base en la física y la biología pierden utilidad cuando se trata de estudiar ciencias como la geología, que usan otros métodos. Hay por tanto que cambiar la manera como se hace historia y filosofía de la ciencia para que puedan aplicarse a la geología, y esta tarea está por hacerse.

Una muestra aleatoria de algunas de las bibliografías de historia de la ciencia aparecidas en Isis e incorporadas en el Cuadro 1 muestra también la poca presencia de la geología. Estas bibliografías recogen referencias tomadas de gran número de revistas y editoriales de todo el mundo, y en el caso de ciencias como la física o la astronomía suelen sumar centenares cada año.
Cuadro 1

Ejemplo de menciones a la geología en Isis (revista oficial de historia de la ciencia, fundada en Bélgica en 1913)

\begin{tabular}{ccc}
\hline $\begin{array}{c}\text { Volumen y } \\
\text { número }\end{array}$ & Año & Menciones \\
\hline 58,5 & 1967 & 10 \\
$59,5 \# 200$ & 1968 & 14 \\
$73, \# 270$ & 1982 & 10 \\
\hline
\end{tabular}

En español tenemos otro ejemplo interesante del papel secundario y derivado que se le ha asignado a la geología. En la colección compilada por Aldo Mieli titulada Panorama General de Historia de la Ciencia el volumen IX está dedicado a la biología y medicina en los siglos XVII y XVIII (Buenos Aires: Espasa-Calpe, 1958). El capítulo XV y último (pp.239-248) se titula "Mineralogía, geología y cristalografía" y después de una breve explicación de por qué se habla de la geología en un volumen dedicado a la biología y medicina - se trata más bien de una disculpa - se nos dice que los comienzos de la geología, en especial estratigráfica y volcánica, deben ubicarse en Francia, debido a los trabajos de Jean Étienne Guettard (1715-1786) y Nicolás Desmarest (1725-1815). Puesto que no hay ninguna mención a la geología en el título de la obra, es por causalidad que uno se entera brevemente de su historia después de largos capítulos sobre la biología y medicina.

Para aclarar esta notable ausencia de una ciencia de importancia reconocida y cuyas raíces se remontan por lo menos a Aristóteles, quizá sea útil sistematizar un poco la historia. Podemos distinguir cuatro etapas en la evolución de las ideas filosóficas sobre la ciencia en general; veremos luego la situación de la geología en cada una de ellas.

(a) En los grandes filósofos griegos aparecen las primeras reflexiones conocidas sobre la naturaleza de la ciencia, entendida ésta como modelo y patrón del conocimiento o bien como única forma válida de conocer. Platón dedicó largas conversaciones de sus diálogos a este tema, que 
Aristóteles retoma con gran detalle en la segunda parte de sus Analíticos. Cuando en la Edad Media autores cristianos, judíos y musulmanes dan forma a la teología, tienen en mente el ideal clásico de la ciencia como conocimiento perfecto e indubitable. Esta visión se prolonga luego en filósofos del siglo XVII y se puede ver hasta en algunos científicos del siglo XIX.

(b) Encontramos luego la exaltación de la ciencia experimental como único modo aceptable de conocimiento, contra las pretensiones de la metafísica y de la teología, que reclamaban para sí no solo la posesión de conocimiento sino además la posición más alta en la jerarquía de las ciencias. Aunque esta reivindicación de la experiencia, observación e inducción se remontan a Francis Bacon en el siglo XVII, es en el XIX cuando se llega a su máxima expresión. Tal es la visión del positivismo de Augusto Comte, pero también y en otra forma muy diferente, del marxismo de la misma época. Comte no era científico, sino filósofo, pero su filosofía consiste en exaltar la ciencia hasta convertirla en religión. Marx partió de su formación filosófica para formular una ciencia nueva, la economía política. Ambos consideran que lo que necesitamos es la ciencia, no la filosofía, aunque por razones y para propósitos diferentes.

(c) En la etapa siguiente son los científicos quienes buscan sólidos fundamentos lógicos y epistemológicos para sus teorías. Este movimiento empieza con Ernst Mach, se prolonga en el Círculo de Viena y llega hasta los autores que se separan de éste usando sin embargo una terminología compartida que se deriva de una formación científica común. Dentro del positivismo lógico, o fuera de éste pero midiendo la distancia que los separa, este grupo de autores llega hasta Thomas S. Kuhn y sus seguidores, particularmente con el famoso ensayo La estructura de las revoluciones científicas (1962, segunda edición ampliada en 1970).

(d) Finalmente, aparecen quienes cuestionan o rechazan toda pretensión de superioridad en la ciencia y la reducen a un fenómeno social siempre contaminado por el poder, la ideología, la lucha de clases o las agendas de grupos opuestos. Puesto que ahora son científicos sociales o filósofos con formación en ciencia social quienes hablan así sobre la ciencia, se afirma a veces con imprecisión pero no mucha exageración que se trata de una venganza de las ciencias sociales sobre las naturales. Aunque con grandes diferencias entre ellos, varios grupos que comparten la actitud crítica y a veces hostil ante la ciencia se ubican en esta época: postmodernos, constructivistas sociales, relativistas, feministas radicales. En muchos de los autores de estas tendencias el aspecto social de la ciencia se vuelve determinante: la ciencia es exitosa únicamente porque así la consideran algunos grupos interesados.

\section{Una ciencia no cuestionada}

Es de notar que la geología apenas aparece en cada una de estas etapas. Hasta el siglo XVIII la razón de esta ausencia parece ser la falta de identidad separada de la geología, englobada dentro de lo que se llamó filosofía natural por lo menos hasta tiempos de Newton. En el periodo de su aparición como ciencia diferente con su propia institucionalidad, entre fines del XVIII y mediados del XIX, es cuando también aparecen algunas reflexiones sobre su naturaleza, sin continuidad durante el gran periodo de la filosofía de la ciencia que empieza con Mach y se prolonga durante la primera mitad del siglo XX. En cuanto a las discusiones posteriores, marcadas por el relativismo postmodernista, el externalismo y el constructivismo social, se nos ocurre que la geología no ha sido blanco de los ataques que han sufrido otras ciencias porque su papel social ha sido menos conspicuo y más aceptado por el público en general. La geología se ha mantenido al margen de las controversias que han rodeado a los químicos, físicos y biólogos, aunque corren el riesgo de verse envueltos en disputas sobre predicción de terremoto, erupciones y deslizamientos, como veremos más adelante.

Basta una breve comparación con las otras ciencias llamadas naturales. La química tuvo que ver con la fabricación de gases mortíferos usados en la guerra de trincheras entre 1914-1918 y en los campos de exterminio durante la Segunda Guerra, así como con las bombas incendiarias que cayeron sobre las grandes ciudades de Alemania y Japón matando a centenares de miles de civiles indefensos. Famosas compañías químicas produjeron el Zyklon-B usado en campos de exterminio nazis, 
así como el napalm y los defoliantes que tanto daño hicieron en Vietnam. En cuanto a la física, sin las teorías de dicha ciencia y sin la intervención personal de famosos físicos es difícil imaginarse la fabricación y uso de las bombas atómicas que destruyeron dos ciudades japonesas en agosto de 1945 . De modo que la física y la química han quedado manchadas por la participación de sus practicantes en la fabricación de armas destructoras de millones de vidas humanas, en su mayoría civiles.

El caso de la biología es diferente, porque los ataques contra ella tienen que ver menos con acciones u omisiones de los biólogos. Esta ciencia se ha visto asediada ante todo por los fundamentalistas que no aceptan la teoría de la evolución por razones religiosas. La disputa sobre los organismos transgénicos, en cambio, es parcialmente una discusión dentro de la ciencia y no solo un ataque externo.

Hasta ahora, en cambio, la geología ha quedado al margen de las controversias surgidas a raíz de la aplicación en armas de destrucción masiva o del choque de opiniones religiosas con teorías científicas. Los fundamentalistas atacan a Darwin, no a Lyell o Hutton. Darwin es un científico ampliamente conocido (aunque no por sus aportes a la geología) mientras los fundadores de la geología son generalmente desconocidos. En esto se parece a la astronomía, que también se ha librado de los ataques de que han sido objeto otras ciencias. También como los astrónomos, los geólogos no pueden experimentar directamente con los objetos que estudian. No pueden mover las placas tectónicas a ver qué ocurre, ni provocar a los volcanes para que hagan erupción.

Lo curioso es que los ataques a la evolución no afecten a la geología. Quienes rechazan el evolucionismo darwinista a veces afirman que la Tierra fue creada hace menos de 5000 años, lo que obviamente pone en entredicho las series temporales de capas y placas que se enseñan en geología. Pero para los creacionistas enemigos de la evolución los villanos son Charles Darwin - en cuanto biólogo - y sus seguidores, no los de Werner, Hutton y Lyell. Cuando los fundamentalistas han logrado que se prohíba la enseñanza obligatoria de la evolución en escuelas de varios estados norteamericanos no han sido consistentes pues no han pedido que se impida la enseñanza de la geología. Para ser lógicos, deberían haber gestionado ante todo la prohibición de la enseñanza de la paleontología, sin cuyo sustento poco puede hacer la teoría de la evolución.

\section{Ni atacada ni defendida}

Si la geología no es controversial, tampoco se han fijado en ella los constructivistas sociales, para quienes toda ciencia es producto de una sociedad determinada y está condicionada por las fuerzas sociales prevalecientes. Mientras para el filósofo analítico el origen de un enunciado es irrelevante para su verdad o falsedad, el constructivista social considera que no podemos separar las teorías de las ciencias de las personas que las proponen o las rechazan. Uno de los más conocidos e interesantes de este grupo es Steve Fuller y uno de los libros más básicos e importantes en esta visión de la ciencia es su obra Philosophy, Rhetoric, and the End of Knowledge (Madison: The University of Wisconsin Press, 1993). En un voluminoso libro que abunda en referencias a la antropología, computación, ecología, economía, educación, etnografía, historia, periodismo, lingüística, física, ciencias políticas, psicología, retórica y sociología, no aparecen la geología ni los geólogos. Tampoco está presente en otra obra posterior e igualmente voluminosa del mismo autor, Thomas Kuhn, A Philosophical History of Our Times (Chicago y Londres: The University of Chicago Press, 2000). Como su título lo indica, el libro entero está dedicado a analizar y criticar el pensamiento de Kuhn, a quien Fuller considera demasiado tímido en su relativización de la ciencia y ambiguo en sus posiciones. De acuerdo con la idea de los constructivistas sociales de que no se puede hacer una separación entre los científicos y sus ciencias, la obra abunda en detalles de la vida personal del autor estudiado que a otros nos parecen irrelevantes. Pero volvemos a la misma constatación: la geología no aparece en la discusión.

Si la geología no ha sido atacada, entonces tampoco hay que defenderla. Entre los defensores de la ciencia en general frente a los ataques relativistas, postmodernos y constructivistas hemos escogido dos autores recientes: Susan Haack y 
Robert Klee. Susan Haack, profesora en Inglaterra y ahora en Miami, es bien conocida por sus libros de lógica y filosofía de la ciencia y se ha convertido en una de las escritoras más apasionadas en defensa de la ciencia. El primer libro suyo que nos parece relevante al respecto es Evidence and Inquiry, Towards Reconstruction in Epistemology (Oxford: Blackwell Publishers,1993), seguido por Manifesto of a Passionate Moderate, Unfashionable Essays (Chicago y Londres: The University of Chicago Press, 1998) y Defending Science within Reason (Amherst, N.Y.: Prometheus Books, 2003). Otra vez es notable la ausencia de una ciencia a la que por lo demás encontramos cada rato en la vida cotidiana: en ninguna de estas obras se ocupa Susan Haack de la geología. Estamos acostumbrados a oír hablar de placas tectónicas, yacimientos de minerales, capas y sedimentos, y sin embargo la ciencia que nos habla de estas cosas ha logrado escapar tanto de los ataques de críticos como de las defensas de amigos. Éstas no han sido necesarias porque aquéllas no se han dado.

Robert Klee, profesor en Ithaca College, publicó en 1997 Introduction to the Philosophy of Science, Cutting Nature at its Seams (Oxford University Press). Además de criticar las tesis típicas del relativismo y constructivismo social, Klee es interesante porque explícitamente trata de corregir el sesgo de la filosofía de la ciencia hacia la física y toma como ejemplo para hacerlo la inmunología, con la que está ampliamente familiarizado. Pues bien: en esta obra tan reciente e importante de nuevo está ausente la geología, ni siquiera mencionada en el índice analítico.

\section{Algunos temas que podrían ser interesantes}

El anonimato tiene sus ventajas, pero también ha evitado que se discutan algunos aspectos interesantes de la geología que podrían ser objeto de la discusión filosófica en el futuro. Nos referiremos únicamente a dos problemas generales: el origen de la geología como ciencia, y su objeto. Luego analizaremos brevemente el caso concreto de la predicción en sismología y de los problemas epistemológicos y éticos que conlleva, a propósito de un caso particularmente sorprendente en Costa Rica.
Para el primero volvamos a la obra de Laudan mencionada arriba, From Mineralogy to Geology. La autora rechaza la opinión prevaleciente sobre la geología, caracterizada por las siguientes tesis:

(a)La geología llegó a ser ciencia a principios del siglo XIX cuando sus fundadores empezaron a ocuparse de desenterrar la evolución de las capas terrestres.

(b) Los fundadores de la geología fueron británicos.

(c) Ellos reemplazaron las especulaciones teóricas con estudios empíricos.

(d) Al hacerlo, liberaron la ciencia de la religión. En vez de leer la Biblia, empezaron a observar las rocas.

Contra estas tesis Laudan argumenta:

(á) La geología era una ciencia ya a fines del siglo XVIII.

(b’) Surgió de la mineralogía, no de la historia de las etapas de la corteza terrestre.

(c') Nació en Alemania con Gottlob Abraham Werner y sus discípulos.

(d’) Werner ya había liberado la geología de las ataduras religiosas.

Lo anterior es tema de una discusión histórica, no filosófica. Determinar quién tiene razón no es tarea del filósofo, sino del historiador. Pero la discusión nos lleva a una pregunta que sí tiene interés filosófico, a saber, ¿cuál es el propósito de la geología? ¿Cuál es el ámbito de los geólogos dentro del conjunto de los científicos? Esto nos lleva a una pregunta más filosófica aún: ¿cuál es el objeto de la geología, y cómo se relaciona este objeto con el de las otras ciencias, sobre todo la física y la química? Hoy nos resulta fácil identificar la geología con rocas y placas tectónicas, y ubicar la sismología dentro de este ámbito de un modo semejante a como la meteorología se relaciona con la física. Las rocas y placas se mueven; la temperatura y humedad cambian. Otra manera de caracterizarlas sería la siguiente: la física se centra en el átomo, la química en la molécula, la biología en la célula y la geología en ciertas clases de elementos, compuestos y procesos. La imprecisión de esta definición salta a la vista. Volver a la etimología del término y decir que la geología es el estudio de la Tierra ayuda menos aún. Por un lado la geografía también estudia la Tierra; por otro, se habla de procesos geológicos en otros planetas. Precisar el objeto es todavía un tema interesante. 
Laudan distingue dos enfoques en el estudio de los objetos de la geología: el histórico y el causal. El primer enfoque busca la sucesión de aspectos del paisaje (cadenas montañosas, ríos, valles,etc.) en diferentes lugares para generalizar las etapas y buscar luego los detalles de cada una en distintos parajes. El enfoque causal se pregunta por qué han ocurrido los cambios en el paisaje, y también trata de generalizar la respuesta de modo que se aplique a cualquier región. El enfoque histórico se centra en el cómo, el causal en el por qué. Ante una formación rocosa, o un derrumbe, nos podemos preguntar cómo se produjo y esto nos lleva a una descripción diacrónica. $\mathrm{O}$ podemos preguntarnos por qué se produjo, y la explicación entonces tiende a ser sincrónica. Aunque el libro reseñado afirma que geólogos diferentes prefieren uno u otro enfoque, probablemente la mayoría los usan en forma conjunta sin pensar en el asunto. Un buen ejemplo es la hipótesis o conjetura (llamada "teoría" en artículos de periódico) de que los sismos en Costa Rica se dan en ciclos de aproximadamente 10 años separados por periodos de poca actividad sísmica que duran más o menos 40 años. Éste es un enfoque obviamente histórico, que sería una inducción si se basara en un recuento de todos los casos conocidos ("en el pasado ocurrió siempre de esa manera, luego en el futuro ocurrirá de igual modo") pero que más bien es una analogía por la limitación de los casos considerados ("éste es un ciclo de sismos que se parece al anterior en la forma como empezó, luego se parecerá también en la forma como terminará"). Es notable que al mismo tiempo los partidarios de esta conjetura añadan una explicación causal, sobre todo cuando se señalan los problemas evidentes de la hipótesis de ciclos: la liberación de energía se ha atrasado en un sector donde las placas chocan, de tal modo que se puede pronosticar la proximidad de un sismo de grandes proporciones. Ni la inducción ni la analogía son argumentos necesarios, pues en ambos las premisas pueden ser verdaderas y la conclusión falsa. Por otra parte, la fuerza de la explicación causal depende de acertar con la causa correcta; de ahí que algunos autores hablen de la causa verdadera como uno de los tipos de explicación en geología.

En la búsqueda de sucesiones temporales de los fenómenos que estudia, la geología se parece a la arqueología, paleontología y algunas ramas de la biología. En este sentido difiere de la física y la química, donde los eventos no se ordenan en cadenas temporales. Esta diferencia se ha expresado en forma exagerada diciendo que en historia $\mathrm{y}$ en las ciencias que establecen sucesiones temporales los hechos nunca se repiten mientras en física y química se repiten constantemente. Hay otra versión de esta distinción, según la cual el físico y el químico pueden repetir sus experimentos cuantas veces quieran mientras el historiador y el geólogo ni siquiera pueden interferir en los eventos estudiados. La visión es exagerada porque la separación no es tan completa (el físico no puede repetir lo que ocurrió en el "big bang"; el geólogo puede hacer réplicas y modelos a escala de algunos procesos), pero apunta en la dirección correcta aunque en forma aproximada.

También es aproximada la afirmación de que la geología es más abstracta que la geografía, incluso cuando ya había nombre para la segunda pero no para la primera. El geógrafo Tolomeo en el siglo II se preguntó sobre el origen del Nilo y lo ubicó en las misteriosas Montañas de la Luna (hoy conocidas como Rwenzori en Uganda), pero siglos antes Aristóteles se había hecho otra pregunta más general, la del origen de cualquier río, y había rechazado la idea de que todos procedían de un enorme y único depósito subterráneo de agua. Estas preguntas no se excluyen entre sí, y por tanto la geología no elimina la geografía: había que verificar si Tolomeo tenía razón, si realmente existen las Montañas de la Luna y dónde están, y si allí nace el Nilo. Una respuesta a estas preguntas tuvo que esperar hasta finales del siglo XIX y principios del XX.

Todavía el filósofo puede entrar en la discusión fijándose en los términos más que en los datos: ¿cuáles leyes generales utiliza el geólogo? ¿Son esas leyes propias de la geología, o más bien de otras ciencias? Al llegar a este tipo de discusión la distinción entre el enfoque histórico y el causal se diluye, lo que muestra que la diferencia no es tan profunda. Cuando uno abre un libro de geología se encuentra enseguida con la fijación de periodos temporales en los que tuvieron lugar los eventos, pero también encuentra explicaciones de por qué ocurrieron y, por tanto, referencia a causas y efectos. La fijación de periodos temporales es común con otras ciencias 
y se basa en principios similares, sobre todo el de uniformidad: los acontecimientos que tuvieron lugar en el pasado obedecieron a las mismas leyes que encontramos en el presente.

En la búsqueda de causas otras ciencias como la física o la química están en el trasfondo, pero el resultado final de su aplicación es típico de la geología. Aunque las ciencias tengan muchas cosas en común, cada una ha adquirido su propia fisonomía y legitimidad. Parte de la diferenciación obedece a la evolución de las instituciones. El papel social de los geólogos es diferente al de otros científicos, pero también al de los ingenieros. Esto no basta para evitar conflictos entre profesiones, sobre todo cuando hay financiación de por medio. La historia de la ciencia y de la tecnología es en buena medida un relato de conflictos, no solo sobre teorías, sino también sobre competencias profesionales.

Además de enfoques generales, el filósofo de la ciencia suele tener una especial debilidad por los tipos de razonamiento frecuentes en cada ciencia, sobre todo los usados por los fundadores. En el caso de la geología Laudan distingue cuatro caminos para la construcción de las teorías propias de esta ciencia: hipótesis, analogía, inducción y causa verdadera. Cada uno de estos tipos se relaciona con el modo de prueba utilizado y con la naturaleza de la teoría formulada. Ninguno de ellos es exclusivo de la geología, pero la preferencia de unos sobre otros y las diferentes combinaciones de métodos difiere en cada ciencia. Mientras la física y la química pueden buscar la confirmación empírica de sus teorías en los experimentos, la geología - al igual que la astronomía- se limita a la observación de procesos y fenómenos con los que no se puede interferir a no ser en escala muy pequeña. Pero la analogía tiene un papel en la geología mayor que en otras ciencias semejantes. En el laboratorio se pueden simular algunos procesos geológicos. El éxito en la predicción de los resultados, sin embargo, solo se puede transferir a la teoría geológica general mediante analogías. Lo que la teoría exitosa predice directamente es que los resultados de laboratorio serán de esta u otra manera, no que la Tierra se haya comportado de esa forma. Esta conclusión solo es indirecta.

Según David B. Kitts en el otro libro mencionado arriba, The Structure of Geology, la meta de la geología es el descubrimiento y verificación de enunciados singulares descriptivos sobre eventos pasados. Pero esto no significa que la geología sea meramente descriptiva, pues los eventos pasados descritos por el geólogo no están simplemente dados en la experiencia, sino que se infieren a partir de enunciados sobre el presente, juntamente con las leyes apropiadas. Esto nos plantea algunas preguntas de segundo orden: ¿se puede aplicar a la geología las nociones de paradigma, anomalía y revolución popularizadas por Thomas S. Kuhn? Si así fuera, ¿se han dado revoluciones en geología? Si en vez de Kuhn nos interesa un filósofo de la ciencia muy diferente, Karl R. Popper, ¿cómo se aplicaría el método hipotético-deductivo en geología? La tentación obvia es decir que este trabajo está por hacerse entre nosotros. Pero existe el peligro de embarcarse en discusiones interminables que no han ayudado gran cosa a otras ciencias. Hay otras corrientes en filosofía de la ciencia y de la tecnología más propias de América Latina, y más prometedoras para nosotros, de las que surgen otras preguntas: ¿cuál es la relación entre geología y tecnología? ¿Cómo relacionar la geología con el desarrollo socio-económico? Estos temas nos parecen más interesantes, aunque aquí solo podamos mencionarlos.

Seleccionemos un problema especial, el de la predicción en geología, y un caso particular de predicción en un país subdesarrollado, Costa Rica.

\section{Predicción de sismos: problemas de un caso particular}

A diferencia de los científicos, que deben olvidarse de sus preferencias al estudiar el objeto de su ciencia, los filósofos colocan en primer término sus valores y conciben sus intervenciones como una defensa de éstos. Por esta razón es frecuente que los filósofos promuevan la crítica de lo que consideran cuestionable. Sirva lo anterior para explicar el tono crítico de lo que sigue.

A la hora de predecir sismos todavía hay poca diferencia entre el experto y el lego. En regiones de alta sismicidad como en la que se encuentra Costa Rica, lo más que podría decir alguien sensato es que en cualquier momento puede ocurrir un terremoto. Si uno estudia la historia de los grandes sismos es difícil encontrar 
patrones a largo plazo : en tiempos recientes se han registrado terremotos en 1822, 1841, 1888, 1910, 1924, 1950, 1955, 1983, 1990, 1991.

La historia que vamos a relatar empieza después de 1983. Con el terremoto del Sábado Santo de ese año se inicia un periodo de intensa actividad sísmica que incluye varios sismos de gran magnitud, en particular el terremoto de Limón el 22 de abril de 1991. Una serie de artículos aparecidos en los periódicos sufren una lenta e interesante transformación: de información sobre lo ocurrido a explicación y predicción de lo que supuestamente está por venir. En la explicación y predicción la fuente es el Observatorio Vulcanológico y Sismológico de Costa Rica (OVSICORI), ubicado en la Universidad Nacional, citado en todos los artículos que hemos podido recolectar en conexión con este tema. Lo que nos interesa es la argumentación, no la exactitud de los datos aparecidos en los periódicos. Respecto de esos datos lo único que podemos hacer es tomarlos como están y analizarlos en el contexto del razonamiento del que forman parte. Adviértase que no hemos encontrado ninguna aclaración por parte de los geólogos y sismólogos citados como fuentes que corrija lo publicado.

El primer artículo que nos interesa apareció en La Nación el 3 de junio de 1990, y empieza con las siguientes palabras: 'Es una hipótesis, "una verdad provisional", a la espera de alcanzar el "status" de irrefutable'. No queda claro cuál es la hipótesis, pues a continuación se mencionan dos cosas diferentes:

(a) Que el enjambre de sismos que han tenido lugar en Puriscal en los días previos se relaciona directamente con el terremoto del 25 de marzo de 1990, con epicentro en Cóbano e intensidad de 6,5 Richter.

(b)Que existen ciclos de sismicidad en el país, de los cuales hay uno que empezó el 5 de octubre de 1950, conocido como terremoto del Golfo de Nicoya, con magnitud 7,7. Este ciclo se extiende hasta el 1 de setiembre de 1955, cuando ocurrió el terremoto de Toro Amarillo. Luego tenemos el que empezó el 2 abril de 1983, terremoto del Sábado Santo y que se extiende hasta el 3 de julio de ese mismo año.

Nos interesa el punto (b) por ser el que se conecta con la predicción que vamos a analizar. Tres meses después, en el extinto semanario Esta
Semana correspondiente a las fechas del 7 al 13 de setiembre de 1990 aparece un artículo titulado "Los temblores son parte del acomodo de la placa Caribe", en el que se cita un informe elaborado por OVSICORI sobre la secuencia sísmica de Puriscal, y se enuncia claramente una parte importante de la hipótesis: "históricamente la actividad sísmica dentro del Valle Central ha estado asociada a ciclos de actividad fuerte a lo largo de la costa pacífica." Luego se mencionan dos de esos ciclos: 1904-1916 y 1941-1950. En el primero se incluyen dos grandes terremotos costeros en Golfito y Papagayo y se dice que generó grandes sismos como el de Cartago de 1910. En relación con el segundo ciclo se dice que los terremotos costeros en Golfito y Nicoya entre 1941 y 1950 generaron una serie de sismos que terminan con el de Bajos del Toro en 1955, de modo que en realidad el ciclo termina en 1955, y no como podría parecer por lo dicho al comienzo. Aunque no se dice en el artículo, los ciclos tienen entonces una duración parecida: 12 años en el primero, 14 en el segundo.

El país quedó conmocionado por el gran terremoto de Limón el 22 de abril de 1991. En los meses y años siguientes quedaría, además, asustado por la predicción de otro gran sismo inminente. La predicción empieza a tomar forma después de abril. Así, el 10 de agosto de ese año el artículo "Golfito fue el inicio de todo" La Nación incluye sobre el título las palabras "Hipótesis de OVSICORI", la que se enuncia en el primer párrafo: el periodo de actividad sísmica se inició el 2 de abril de 1983 con un terremoto en el Golfo Dulce de magnitud 7,1 y la ola "posiblemente concluirá en 1993". Luego se mencionan los ciclos ya formulados antes (1904-1916 y 1941-1955) y se añade claramente una nueva idea: cada periodo se inicia en el Golfo Dulce y concluye en el Pacífico Norte. Para que esta afirmación se cumpla en el segundo ciclo se menciona un terremoto de Sámara con magnitud 7,7 Richter con el que se cierra el periodo, aunque no se dice la fecha. Con el subtítulo de "La teoría" se procede a continuación a una generalización: "cada 40 años se produce un ciclo de actividad fuerte a lo largo de la costa pacífica" y el artículo termina , en redacción confusa , con la famosa predicción : "esta inducción científica, de resultar cierta, deberíamos 
esperar [sic] a futuro la ocurrencia de un evento importante frente a las costas de Nicoya, con fecha aproximada de 1993, a fin de cerrar el ciclo iniciado con el Terremoto de Golfito en 1983".

Tenemos ya todos los elementos de la "hipótesis" o "teoría" y una predicción con fecha clara. Nótese que se habla de "inducción científica" cuando en realidad se trata de una analogía. Con solo dos ciclos estudiados, la pregunta obvia es qué pasó en épocas anteriores, en los miles de años de actividad sísmica en esta parte del globo. La analogía funciona de otra manera: puesto que A y B se parecen en varios aspectos, también se parecerán en otros.

Llegó y acabó 1993 sin que se produjera el sismo pronosticado. Pero los pronosticadores no se desalentaron. Todavía en 1998 se seguía hablando del terremoto de Nicoya como algo que podría ocurrir en cualquier momento. En $L a$ Nación del 19 de abril de 1998, p. 6A, leemos "En agosto de 1996, [un] especialista de esa institución [OVSICORI], afirmó a este diario: "No se discute si va a ocurrir un terremoto o no (...) Todo indica que sî".

Escribimos estas líneas en el 2006 y debe quedar claro que la predicción basada en la conjetura de los ciclos no se cumplió y que, por tanto, la hipótesis misma está refutada. Incluso si en el futuro próximo se presentara un terremoto en la zona indicada, ese terremoto no sería el pronosticado según la hipótesis de 1991, pues aquella conjetura hablaba de un ciclo de aproximadamente 10 años que había empezado en 1983 y el cual terminó hace ya 13 años. La "teoría" de ciclos sísmicos que duran más o menos 10 años separados por periodos de 40 años, que empiezan en el Pacífico Sur y terminan en el Pacífico Norte de Costa Rica está, por tanto, refutada y los intentos por resucitarla son hipótesis ad hoc. Sin embargo, los artículos sobre el gran sismo de Nicoya han seguido apareciendo por lo menos hasta 2005, y ahora OVSICORI ha ampliado el periodo entre ciclos a 50 años (La Nación, 8/5/2005,17A). No hemos visto que lo haya hecho, pero lógicamente también tendría que ampliar la duración de cada ciclo: en 2006 tendría que ser de 23 años, y no de 10 ó 12 como se decía. Esto parece encajar con el hecho de que en 1998 la fecha se había ampliado aún más: "Los habitantes de la península de Nicoya han escuchado reiteradamente sobre la hipótesis de un sismo de magnitud 7,4 grados en la escala Richter, que ocurriría a lo sumo en 2009" ("Un terremoto...jbah!" en La Nación, 19/4/98,6A). En un amplio artículo en el semanario Universidad correspondiente a la semana del 7 al 13 de julio de 1999 se señalan los daños causados a la economía local con la manera como OVSICORI manejó el asunto. Sin embargo, poco después apareció en La Nación del 25/10/99 el titular "Monte marino amenaza a Nicoya", como una nueva causa para el sismo que se aproxima, y un nuevo motivo de temor para los habitantes de la Península. Ya en el 2004 OVSICORI había vuelto a la carga, esa vez con datos en cuanto a la probabilidad de licuefacción en una zona de aproximadamente 52.679 hectáreas comprendida entre Puntarenas y Santa Cruz ("Aumenta riesgo sísmico en la Península", La Nación, 12/1/2004,4).

Es importante analizar lo ocurrido con esta predicción basada en una conjetura. Las siguientes observaciones nos parecen pertinentes:

(a) La periodización hecha por OVSICORI dejaba por fuera un sismo tan importante como el de Orotina en 1924 del cual se dice: "Es, a juicio de los especialistas, el peor sismo de cuantos han afectado a nuestro territorio" (La Nación ,3/6/1990, artículo titulado "Sismos son consecuencia de Cóbano"; la fuente de este artículo es OVSICORI, hasta donde podemos ver). Si uno de estos ciclos empezó en 1904, si hay un periodo de aproximadamente cuarenta años entre ciclos y si cada ciclo dura cerca de diez años, el anterior tendría que haber empezado alrededor de 1854. Pero en esta periodización no encaja el gran terremoto del 2 de setiembre de 1841, que destruyó la ciudad de Cartago y causó graves daños en pueblos aledaños. Además, se supone que los sismos en el Valle Central tienen relación con esos ciclos en el Pacífico, tal como se dijo originalmente sin que se haya dicho lo contrario en ningún momento.

(b) A partir de dos ciclos, se concluía que tendría lugar otro con características semejantes. ¿Qué había ocurrido antes del primer ciclo analizado en la hipótesis? El territorio nacional se ha visto afectado por sismos desde hace muchos siglos, mientras que la hipótesis de los ciclos tenía en cuenta una época de muy corta duración. 
(c) Al hablar del tercer ciclo (1983-1993) que terminaría con un sismo frente a las costas de Guanacaste, se omitía el detalle de que ya había ocurrido uno dentro de dicho ciclo en esa zona, a saber, el de Cóbano del 25 de marzo de 1990. Según el artículo titulado "40 mil millones en pérdidas" (La Nación, 26/8/91,5A) este terremoto fue de 6,5 grados en la escala Richter y causó numerosos daños en viviendas, escuelas y edificios. Cóbano no está muy lejos de Sámara, lugar frente al cual habría tenido lugar el sismo fuerte "que alcanzó una magnitud de 7,7 grados en la escala Richter" y con el cual supuestamente concluyó el segundo ciclo considerado, el de 1941- 1955 según el artículo en La Nación del 10/8/91, 7A, ya mencionado arriba. En dicho artículo, por cierto, no se dice cuándo ocurrió ese gran terremoto de Sámara con el que se habría cerrado el ciclo 1941-1955, ni aparece ese sismo en la lista de terremotos que encontramos en La Nación del 3/6/90. Más aún: aunque se trata de la lista de grandes terremotos en Costa Rica a partir de 1910, ninguno de los incluidos en ella llega a la magnitud 7,7 Richter.

Pero la confusión aumenta cuando uno lee los artículos de periódicos de esta época. Hubo un terremoto en Sámara en 1978 con magnitud 7,3 según lo mencionado en La República, 19/4/93, página 19 , pero obviamente no encaja en los ciclos sobre los que se basa la predicción. Suponemos que este terremoto de Sámara es el mismo que aparece con el nombre de Nicoya en el recuadro aparecido en La Nación del 16/5/94,p.8A, dentro del artículo titulado “¿Falta aún otro terremoto?" Tal recuadro ofrece una exposición gráfica de los ciclos pero las fechas son confusas: del primer ciclo se dice que va de 1904 a 1912 pero que termina en 1916 , el segundo cubre de 1941 a 1950 pero se incluye un evento de 1955 y del tercero se dice que empezó en 1983 pero que no se sabe cuándo termina. Tal vez el terremoto de Sámara sea el mismo "terremoto del Golfo de Nicoya, que tuvo una magnitud de 7,7" del 5 de octubre de 1950, del que se habla en La Nación, 3/6/90. Difícilmente podría ser otro, pero Sámara y el Golfo de Nicoya no son lo mismo. Además, parece extraño que un terremoto de semejante magnitud no figure en varias listas de grandes terremotos en el territorio nacional aparecidas en estos artículos de periódico: ni en la que aparece en La Nación, 3/6/90 ni en otra más detallada incluida como recuadro dentro del artículo "Periodo sísmico no ha concluido", en La República del 19/4/93, p.19.

(d) A pesar del uso frecuente de términos y expresiones como "probable" y "de resultar cierta (...)" (La Nación, 10/8/91,7A), "esto es solo una teoría" (La Nación, 8/3/92,5A), la insistencia en la probabilidad obviamente produce efectos en la vida cotidiana de los habitantes de la zona, así como consecuencias económicas debido al alejamiento de los turistas. La insistencia en afirmar una proposición con el efecto de producir otro resultado diferente es típica de la falacia de énfasis. El énfasis sugiere que la proposición enfatizada implica algo más. Si se repite cada rato que hay una alta probabilidad de que ocurra algo, el resultado - que puede ser no intencional-es sugerir que hay certeza de que ocurrirá.

(e) La afirmación de que se trata de probabilidad no encaja con la fijación de fechas, que supone certeza: "con fecha aproximada de 1993", "a lo sumo en 2009" son expresiones textuales tomadas de artículos citados.

(f) Según la fecha límite ahora fijada por OVSICORI para el sismo de Nicoya, éste tendrá lugar a más tardar en 2009. Para esta predicción se hace referencia a datos, equipos, estudios, análisis, instrumentos, visitas de geólogos extranjeros y de un barco oceanográfico. Ahora bien, podemos atrevernos a formular el siguiente pronóstico: antes del año 2051 habrá un sismo fuerte en algún punto en el Pacífico Norte de Costa Rica frente a la península de Nicoya. Si tiene lugar el sismo pronosticado por OVSICORI, se cumplirá no solo su predicción sino también la nuestra. Si el sismo tiene lugar después de 2009 pero antes de 2051, no se habrá cumplido la predicción de OVSICORI pero sí la nuestra. En cualquiera de los dos casos se cumple esta nueva predicción, que no se basa en ninguna conjetura de ciclos, ni en datos de instrumentos, ni en opiniones de científicos visitantes, sino en una simple conjetura basada en el conocimiento ordinario.

Para terminar con este tema: todavía no se puede pedir a los geólogos que hagan predicciones de terremotos. Si las hacen, corren el riesgo de provocar la incredulidad. 


\section{CONCLUSIÓN}

Más allá de los aspectos anecdóticos de una predicción fallida, queda el tema general de la relación entre explicación y predicción planteado en la filosofía de la ciencia y que podría aplicarse a la geología: ¿Son simétricas la explicación y la predicción? ¿Es posible predecir en todas las ciencias, o hay algunas en las que únicamente se puede explicar? En este artículo hemos constatado una situación peculiar de la geología, que goza de un alto grado de inmunidad frente a las críticas contra la ciencia tan comunes en nuestros días. ¿Hay alguna explicación para esta situación? Otros temas interesantes en los que aún queda mucho por explorar tienen que ver con la lógica de la geología (validez o invalidez de sus argumentos), la epistemología de dicha ciencia (verdad o falsedad de sus enunciados) y la ética de la actividad científica (bondad o maldad de las acciones).

\section{REFERENCIAS}

FEIGL,H. \& SELLARS, W. (comp.), 1949: Readings in Philosophical Analysis.626 págs.- Appleton-Century-Crofts,Inc, Nueva York.

FEIGL, H, \& BRODBECK, M. (comp.), 1953: Readings in the Philosophy of Science.811 págs.- Appleton-Century-Crofts, Inc, Nueva York.

FULLER, S., 1993: Philosophy, Rhetoric , and the End of Knowledge.- 421 págs.- Madison: The University of Wisconsin Press.

KUH, T., 2000: A Philosophical History of our Time.- 471 págs.- The University of Chicago Press, Chicago.

GEIKE, A., 1962: The Founders of Geology.- 416 págs. Dover Publications, Nueva York.
GONZÁLEZ VÍQUEZ, C., 1910 [facsímil publicado en 1994]: Temblores, terremotos, inundaciones y erupciones volcánicas en Costa Rica 1608-1910.- 239 págs. Editorial Tecnológica de Costa Rica, Cartago.

GREENE, M., 1985: The History of Geology.Osiris, 1: 97-116.

HAACK, S., 1993: Evidence and Inquiry, Towards Reconstruction in Epistemology.- 259 págs. Blackwell Publishers, Oxford.

HAACK, S., 1998: Manifesto of a Passionate Moderate, Unfashionable Essays.- 223 págs. The University of Chicago Press, Chicago.

HAACK, S., 2003: Defending Science within Reason.- 411 págs. Prometheus Books, Amherst, NuevaYork.

KITTS,D.B., 1977: The Structure of Geology.- 180 págs. Southern Methodist Press, Dallas.

KLEE, R., 1997: Introduction to the Philosophy of Science: Cutting Nature at Its Seams.258 págs. Oxford University Press, Nueva York-Oxford.

LAUDAN, R., 1987: From Mineralogy to Geology: The Foundations of a Science, 1650-1830.278 págs. Chicago University Press.

MIELI, A. (comp.), 1958: Panorama General de Historia de la Ciencia, tomo IX Biología y Medicina en los siglos XVII y XVIII por Desiderio Papp y José Babini.- 252 págs. Espasa-Calpe Argentina, Buenos Aires.

NAGEL, E., 1961: The Structure of Science, Problems in the Logic of Scientific Explanation.- 618 págs. Harcourt, Brace and World,Inc. Nueva York. 
POPPER,K.R.,ADORNO,T.W.,DAHRENDORF, R., HABERMAS, J., 1978: La lógica de las ciencias sociales.- 88 págs. Editorial Grijalbo , México, D.F.

SCHLICK, M., 1925: Philosophy of Organic Life.- En: FEIGL, H. \& BRODBECK, M. (comp.), 1953: Readings in the Philosophy of Science.- Appleton-Century-Crofts, Inc., Nueva York: 523-536.

SCHLICK, M., 1935: On the Relation Between Psychological and Physical Concepts.- En: FEIGL, H. \& SELLARS, W. (comp.), 1949: Readings in Philosophical Analysis. AppletonCentury-Crofts, Inc., Nueva York: 393-407. 